\title{
Marine Circular Economy towards Post-Disaster Reconstruction for Sustainability: Experiences in a Small Coastal Town of Northeast Japan
}

\author{
Ai Tashiro ${ }^{1}$, Yuta Uchiyama ${ }^{1}$, Ryo Kohsaka ${ }^{1}$
}

\begin{abstract}
The wealth of evidence on the conservation effects of development and utilization of ocean, whereas what shapes behavioral change remains unknown, particularly of fishermen. Few previous studies incorporate measuring changes in fishermen's behavior. This study aims to investigate what changed behavior and perception of fishermen toward marine circular economy in Japan. We selected a unique case of marine-linked practical change of fishermen's behavior. Our study area, Minamisanriku town in Miyagi Prefecture, was affected in the 2011 earthquake and tsunami. The town incorporated marine circular economy (MCE) into post-disaster reconstruction project: Fishermen reduced farming facilities to one-third of that before the disaster and the quality of oyster improved. To indicate the determinant facts of the practical change, we used ordered probit model for fishermen recognition on the effectiveness of integrating marine conservation into post-disaster reconstruction. Our main finding was different recognition level existed amongst fishermen. It was associated with branch affiliation and hope level for the sustainable fishing culture by the next generation. This study provides understanding a tangible and measurable change in recognition can be bridge the gap between idea and practice. This study suggests horizontal collaboration among between branches would lead to marine conservation attitude through community-based postdisaster reconstruction.
\end{abstract}

Keywords: Marine circular economy, fishermen's recognition, post-disaster reconstruction, Japan

\section{Introduction}

The importance of developing marine life and protecting the ocean has attracted wide attention globally. In contrast to the wealth of evidence on the conservation effects of development and utilization of oceans ( $\mathrm{Lu}, 2014)$, much less is understood about what shapes behavioral change, particularly marine ecosystem conservation projects of fishermen. Previous studies have provided ample documentation of the impacts of marine ecosystem conservation projects (Bianchi et al., 2013; Pendleton et al., 2012; Worm \& Lenihan, 2014). However, almost none of them were able to be incorporated the measurement of changes in fishermen's behavior. Thus, it remains unclear whether the marine ecosystem project succeeded because of any changes in behavior through the project activities or whether outside factors among fishermen already pre-date their experience of a disaster. To assess whether a project would succeed or not, there must be a method for the measurement of behavior change. A successful behavior change project should identify a specific type of conservation and awareness, and a specific behavior to target, with a clear strategy as to how to address and measure the particular behavior. 
One notable exception is a study by Dyer and Poggie (2005) on the management of marine resources in Northeastern U.S. fisheries, focusing on the integrated impacts on users and user impacts on resources. In this article, authors suggest that a more holistic approach integrating human capital into the management of large marine ecosystems can improve the speed of recovery from marine resource disasters and lessen the overall negative impacts of regional natural resource disasters on populations of users. However, this article focused only on the large-scale natural resource community and did not focus on the small-scale one. In addition, this article remains unclear on the specific behavior and awareness change of fishermen after a disaster. We are unaware of studies that have focused on small-scale marine resource communities, particularly fishermen's behavior.

To address these limitations, we considered a unique case of marine-linked human ecosystem and change in fishermen's behavior. Our study area, Minamisanriku town in Miyagi Prefecture, was affected by the 2011 Great East Japan Earthquake and Tsunami. The town has been historically famous for aquaculture (e.g., oyster, salmon, and seaweeds). Aquaculture in the area dates back to 1899 and has withstood several tsunamis over centuries. However, most of the aquaculture facilities along this area and cultured rafts were swept away by the tsunami in 2011. Before the tsunami, smallholders ran intensive family farms on small sea surfaces with many rafts that were overcrowded and unsustainable. Starting over provided an opportunity to introduce responsible practices. To this end, the local fisheries cooperative (JF) members joined forces with the World Wildlife Fund Japan to revive the seabed using best practices, including reducing production outputs by a third annually to establish a thriving and balanced base of aquaculture production (ASC, 2016).

What promoted fishermen's behavior change after the disaster? The factors remain unknown. This article aims to investigate what farming practice changes recover the fragile ecosystem and what type of fishermen's recognition of marine conservation and social practices contribute to post-disaster marine reconstruction.

\section{Review}

Overfishing causes marine degradation at a pace that exceeds the ability of individuals, communities, and governments to adapt. Globally, the marine ecosystem paradigm is emerging as a dominant approach to marine resources (Worm \& Lenihan, 2014). For example, resource managers and social scientists are concerned about the degradation of the world's seascapes. In general, natural resource management of outcomes by human environments and communities has fallen behind (Kay, 2006). They suggest focusing on marine ecosystems as a way to recover ecosystem health and to ensure sustainable utilization of marine resources. To approach such changes, marine circular economy (MCE) is regarded as a subsystem of the ecological, social, and economic compound system (Lu, 2014). MCE's development could ensure sustainable development against the marine ecological crisis (Qu, Tsai, Tang, Xu, \& Dong, 2016). In 2017, the World Bank published a report on the long-term benefits of the sustainable use of marine resources for less developed small islands and coastal countries ( $\mathrm{Qu}$ et al., 2016). However, it is an urgent issue not only for such less developed countries but also for developed coastal countries including Japan, which are prone to natural resource 
disasters and insufficiency of MCE. Most previous empirical studies on MCE inadequately captured actual local community practices (Ahmed \& Glaser, 2016;. Thompson, Clubbe, Primavera, Curnick, \& Koldewey, 2014). The innovative approach to marine conservation has led to the recovery of the fragile marine ecosystem. Hence, this study considered a case study of developing community-based reconstruction with MCE to measure what determined fishermen's practical behavioral change after the disaster, focusing on human environmental interface, both at individual fisheries level and at a community level.

\section{Methods}

\subsection{Study Population and Design}

The East Japan Earthquake and Tsunami struck on March 11, 2011. Minamisanriku town, the field site for the present study, is a coastal municipality in Miyagi Prefecture in Japan (Fig.1). A total of 832 people lost their lives or have been missing in Minamisanriku, while $6.1 \%$ of the land mass was inundated. As of June 2018, the total population was approximately 12,300 in this town, among whom $23.7 \%$ were fishermen. In Minamisanriku town, $80 \%$ of the total area is forest, and forest resources and marine resources are linked; the town has longstanding traditions associated with forest and marine resource management that allow sustainable use of natural resources.

To determine fishermen's behavioral change and recognition of sustainable marine production, we conducted a mail survey and sent 400 questionnaires to fishermen in two fisheries co-operative branches: the Shizugawa branch $(n=200)$ and the Utatsu branch $(n=200)$. The survey was conducted from January 25 to February 21, 2018, during which period the questionnaires were completed and sent back to the authors. The questionnaires inquired about demographics as well as marine and forest management, and awareness of conserving them. The response rate was 45.0\% (180/400): Shizugawa, $\mathrm{n}=84$ and Utatsu, $\mathrm{n}=96$. From the 180 responses, we further excluded six because of invalid sex and age, leading to 174 valid responses. A detailed flow chart of the participant selection is presented in Fig. 2 (respondents' flow).

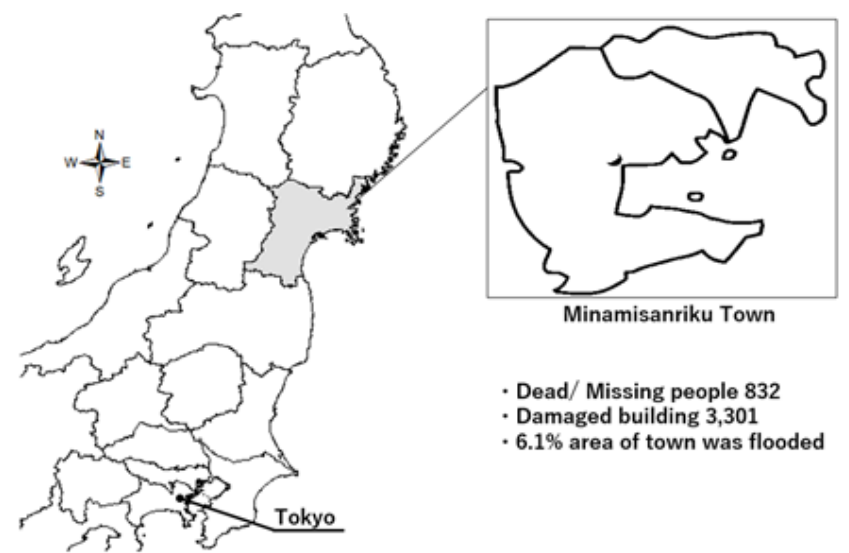

Fig. 1. A map of Minamisanriku Town. (Minamisanriku is a coastal municipality in Miyagi prefecture in Japan.) 


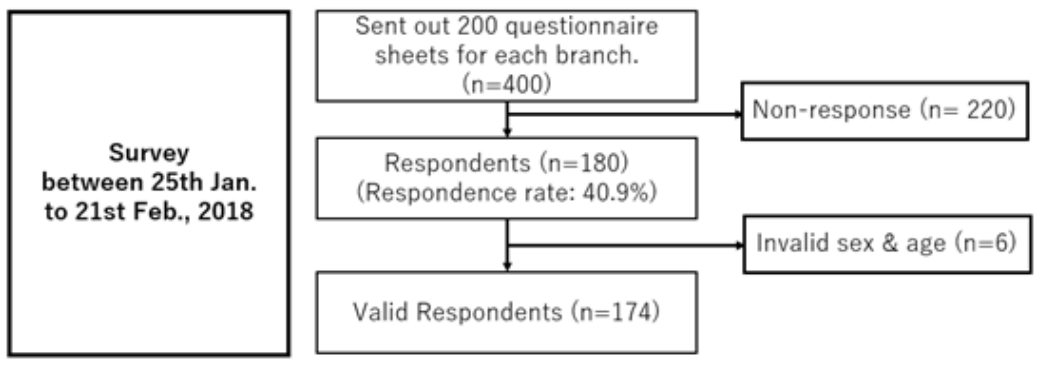

Fig. 2. Respondents' flow in the 2018 survey

\subsection{Independent Variable}

In the survey, each respondent was asked about the recognition of Effectiveness: "Do you think that the reconstruction project is effective in conserving marine resources?" The responses were based on a five-point Likert scale, from never (1) to very effective (5).

\subsection{Covariates}

The demographic characteristics (age, years of education, living with family, period of residence, land holder, Japan Agricultural Cooperative (JA), and affiliation branch), management activity (afforestation experience, forest management, and marine management), and hope (fishermen's hope for a sustainable culture by the next generation) are summarized in Table 1. The average and standard deviations of change in Effectiveness are also shown in Table 1.

\subsection{Statistical Analysis}

We used an ordered probit (OP) model to examine the association between the respondents' characteristics and change in the effectiveness scale, incorporating marine resource conservation into post-disaster reconstruction. Changes in the effectiveness scores were entered as ordered variables. The multivariate adjusted results were expressed as non-standardized coefficients with 95\% confidence intervals (CI) in Table 2. All the analyses were performed with Stata 14.0. Significance was defined as a 2 -sided $\mathrm{P}$ value $<0.05$. We also calculated the predicted probabilities after OP analysis: we estimated the probability of the outcome of each variable (Pr.), setting a predictor to a specific value: branches and hope (Table 3).

Table 1: Main characteristics and effectiveness scale among the study respondents $(\mathrm{N}=174)$

\begin{tabular}{ccccc}
\hline & $\mathrm{n}$ & $(\%)$ & \multicolumn{2}{c}{ Effectiveness } \\
\cline { 4 - 5 } & & & Average & SD \\
\hline Age (years old) & 2 & 1.1 & 2.5 & 0.7 \\
$20-29 \mathrm{y}(=1)$ & 2 & 0.1 & 1.5 & 0.7 \\
$30-39 \mathrm{y}(=2)$ & 20 & 11.3 & 2.3 & 0.9 \\
$40-49 \mathrm{y}(=3)$ & 44 & 24.9 & 2.0 & 0.9 \\
$50-59 \mathrm{y}(=4)$ & 31 & 18.1 & 1.9 & 0.9 \\
$60-64 \mathrm{y}(=5)$ & 58 & 35.6 & 2.2 & 0.9 \\
$65-74 \mathrm{y}(=6)$ & 17 & 7.9 & 2.0 & 0.6 \\
75 y or more $(=7)$ & & & &
\end{tabular}




\begin{tabular}{|c|c|c|c|c|}
\hline & \multirow[t]{2}{*}{$\mathrm{n}$} & \multirow[t]{2}{*}{$(\%)$} & \multicolumn{2}{|c|}{ Effectiveness } \\
\hline & & & Average & $\mathrm{SD}$ \\
\hline \multicolumn{5}{|l|}{ Education (years) } \\
\hline$<6(=1)$ & 1 & 0.6 & 2.0 & 0.0 \\
\hline $6-9(=2)$ & 86 & 50.6 & 2.0 & 0.8 \\
\hline $10-12(=3)$ & 77 & 44.4 & 2.1 & 0.8 \\
\hline 13 or more $(=4)$ & 8 & 4.5 & 2.0 & 1.2 \\
\hline \multicolumn{5}{|l|}{ Living with family } \\
\hline Yes $(=1)$ & 175 & 98.3 & 2.1 & 0.8 \\
\hline No $(=0)$ & 3 & 1.7 & 1.0 & 1.0 \\
\hline \multicolumn{5}{|l|}{ Period of residence } \\
\hline Average (standard deviation) & 24 & 27.6 & & \\
\hline \multicolumn{5}{|l|}{ JA member } \\
\hline Yes $(=1)$ & 124 & 74.2 & 2.1 & 0.9 \\
\hline No $(=0)$ & 43 & 25.8 & 2.1 & 0.9 \\
\hline \multicolumn{5}{|l|}{ Land holder } \\
\hline Yes $(=1)$ & 153 & 91.1 & 2.1 & 0.8 \\
\hline No $(=0)$ & 15 & 8.9 & 2.1 & 1.0 \\
\hline \multicolumn{5}{|l|}{ Afforestation } \\
\hline Yes $(=1)$ & 57 & 32.8 & 2.1 & 1.0 \\
\hline No $(=0)$ & 117 & 67.2 & 2.0 & 0.8 \\
\hline \multicolumn{5}{|l|}{ Hope for sustainable fishery } \\
\hline Don't hope $(=1)$ & 8 & 4.7 & 2.4 & 1.3 \\
\hline Rather don't hope $(=2)$ & 22 & 12.9 & 2.6 & 1.1 \\
\hline Neither $(=3)$ & 42 & 24.6 & 2.1 & 0.8 \\
\hline Rather hope $(=4)$ & 66 & 38.6 & 2.0 & 0.7 \\
\hline Hope $(=5)$ & 33 & 19.2 & 1.9 & 0.7 \\
\hline \multicolumn{5}{|l|}{ Forest management activity } \\
\hline Yes $(=1)$ & 101 & 69.2 & 1.9 & 0.8 \\
\hline No $(=0)$ & 45 & 30.8 & 2.8 & 0.7 \\
\hline \multicolumn{5}{|l|}{ Marine management activity } \\
\hline Yes $(=1)$ & 111 & 74.5 & 1.9 & 0.8 \\
\hline No $(=0)$ & 38 & 25.5 & 2.8 & 1.2 \\
\hline \multicolumn{5}{|l|}{ Branch } \\
\hline Shizugawa (=1) & 81 & 46.8 & 2.0 & 0.8 \\
\hline Utatsu $(=0)$ & 93 & 53.2 & 2.1 & 0.9 \\
\hline
\end{tabular}

\section{Results}

Among the respondents, 106 (61.6\%) fishermen were over the age of 60 years and most of them were living with families, as presented in Table 1 . The average residence period was 24 years. Uniquely, 153 (91\%) fishermen reported having private land and $124(74.2 \%)$ respondents were members of JA. From these results, it can be observed that the fishermen associated their lives as half farming and half fishing. In addition, $57(32.8 \%)$ respondents had experience of afforestation and $101(69.2 \%)$ were interested in forest management activities. They were involved not only in fishing culture but also in forest management.

Table 2 shows the multivariate adjusted associations between demographic 
characteristics and the effectiveness scale of conserving marine resources for postdisaster reconstruction in the OP model. In this model, the hope scale of sustainable marine resource management for the next generation was significantly associated with the recognition of the effectiveness on conserving marine resources for reconstruction: 0.36 points (95\% CI: 0.01, 0.72, $\mathrm{p}=0.046)$. Branch affiliation was also statistically and significantly associated with the recognition of the effectiveness of conserving marine resources for reconstruction: 0.82 points (95\% CI: $0.06,1.57, \mathrm{p}=0.034)$. There was no statistical significance in the relationship between demographic characteristics and the effectiveness score $(\mathrm{p}>0.05)$.

Table 2: Marginal effects of OP model setting predictors to effectiveness score by hope and branch variable

\begin{tabular}{lcccc}
\hline & Coefficient & SE & $95 \%$ CI & \\
\hline Age & 0.05 & 0.15 & {$[-0.25,0.35]$} & 0.74 \\
Education & -0.25 & 0.35 & {$[-0.94,0.44]$} & 0.48 \\
Period of residence & 0.00 & 0.01 & {$[-0.01,0.01]$} & 0.96 \\
Living with family & -0.48 & 1.12 & {$[-2.67,1.71]$} & 0.67 \\
JA member & 0.13 & 0.45 & {$[-0.74,1.01]$} & 0.77 \\
Land holder & 0.09 & 0.65 & {$[-1.18,1.37]$} & 0.89 \\
Hope sustainable fishery & 0.36 & 0.18 & {$[0.01,0.72]$} & 0.046 \\
Afforestation & -0.23 & 0.39 & {$[-0.99,0.53]$} & 0.55 \\
Forest management activity & 0.59 & 0.31 & {$[-0.01,1.19]$} & 0.06 \\
Marine management activity & 0.45 & 0.34 & {$[-0.22,1.13]$} & 0.19 \\
Branch affiliation & 0.82 & 0.39 & {$[0.06,1.57]$} & 0.034 \\
/cut1 & -0.2 & 2.01 & {$[-4.15,3.74]$} & \\
/ cut2 & 2.2 & 1.84 & {$[-1.41,5.81]$} & \\
/ cut3 & 4.25 & 1.87 & {$[0.58,7.91]$} & \\
/ cut4 & 6.93 & 1.93 & {$[3.15,10.70]$} & \\
\hline Ordered logistics regression & \multicolumn{3}{c}{} & \\
LR chi2=34.39. Prob $>$ ch=0.000. Pseudo R2=0.115. & & & \\
\hline
\end{tabular}

Table 3 shows the marginal effects of factors identified in the OP model. We applied the statistically significant variables "hope" and "branch affiliation" in the results of the model. The estimated results are presented in Table 3. The marginal coefficients illustrate the change in occurrence probability of the effectiveness score by a unit increase in the input variables "hope" and "branch affiliation," keeping other factors at their mean values. In Fig. 3, a positive marginal coefficient of a variable for a particular level reflects that the probability of the level will increase by a value equal to the coefficient with a unit increase in hope level, and vice versa. Fig. 3 concluded that red lines, which indicate "very effective," will increase the effectiveness score by 8, 11, 16, 21, and 28 percent. In contrast, the broken red line represents "effective," and we can observe that regardless of the hope level, the probabilities will gradually increase and be higher than the other effectiveness scale line. In contrast, the gray line and broken blue line indicate "neither" and "not effective," respectively. In these lines, an increase in the hope level will decrease the effectiveness score. The blue line indicates "never" and it shows any statistical significance. In Table 3, the probabilities of effectiveness on branch affiliation, the proportion of "effective" was the highest probabilities in both Shizugawa and Utatsu. The 
probability of "effective" and "very effective" in Shizugawa was higher than that of Utatsu by 2 and 12 percent, respectively.

Table 3: Marginal effects of the OP model, setting predictors of effectiveness score by the variables "hope" and "branch"

\begin{tabular}{lcccc}
\hline & Coefficient & SE & $95 \%$ CI & p-value \\
\hline Age & 0.01 & 0.15 & {$[-0.25,0.35]$} & 0.74 \\
Education & -0.25 & 0.35 & {$[-0.94,0.44]$} & 0.96 \\
Period of residence & 0.00 & 0.01 & {$[-0.01,0.01]$} & 0.67 \\
Living with family & -0.48 & 1.12 & {$[-2.67,1.71]$} & 0.77 \\
JA member & 0.13 & 0.45 & {$[-0.74,1.01]$} & 0.89 \\
Land holder & 0.09 & 0.65 & {$[-1.18,1.37]$} & 0.045 \\
Hope sustainable fishery & 0.36 & 0.18 & {$[0.01,0.72]$} & 0.046 \\
Afforestation & -0.23 & 0.39 & {$[-0.99,0.53]$} & 0.55 \\
Forest management activity & 0.59 & 0.31 & {$[-0.01,1.19]$} & 0.06 \\
Marine management activity & 0.45 & 0.34 & {$[-0.22,1.13]$} & 0.19 \\
Branch & 0.82 & 0.39 & {$[0.06,1.57]$} & 0.034 \\
/ cut1 & -0.20 & 2.01 & {$[-4.15,3.74]$} & \\
/ cut2 & 2.20 & 1.84 & {$[-1.41,5.81]$} & \\
/ cut3 & 4.25 & 1.87 & {$[0.58,7.91]$} & \\
/ cut4 & 6.93 & 1.93 & {$[3.15,10.70]$} & \\
\hline Not & im & &
\end{tabular}

Note: *indicates $p<0.05$. Pr. represents the predicted probability of the effectiveness score in each hope level.

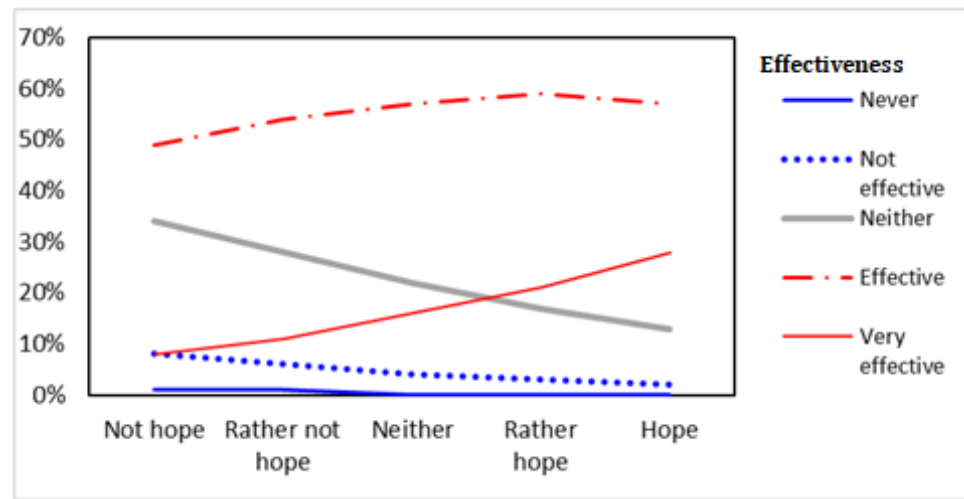

Fig. 3. Marginal effects of the effectiveness score amongst respondents for the OP model by hope level

\section{Discussion}

This study investigated the reasons that changed fishermen's behavior in fishing culture reconstruction in the MCE in Japan. MCE development is the premise and approach of securely constructing a marine ecology in disaster affected areas (Mei \& Yin, 2009). However, this not only focuses on the system but also seeks to determine the facts that promote the actors' behavior change that should be key to creating a project design (Dyer \& Poggie, 2005; Wong \& Candolin, 2015). Based on our knowledge, this is the first study to utilize information on post-disaster reconstruction to examine and 
measure changes in behavior and perception among local fishermen's communities in Japan. This study found a difference in the effectiveness recognition among fishermen for post-disaster reconstruction concerning marine conservation even in the same town. It was related to branch affiliation and hope level for the sustainable fishing culture of the next generation. Fishermen were succeeding not only by rebuilding their community but also by improving the marine environments. Reducing farming facilities to one third contributed to improving the quality of the oysters; currently, it takes one year until harvest, while it took three years before the disaster. This study's insight is an important example of the resilience of the fisheries community.

The Marine Debris Program (2017) recently identified how to address and measure the fishermen's behavior that promotes a clearing strategy for recovery from a disaster. For policy-making, the difference in recognition among fishermen would interfere with marine reconstruction support (World Bank and United Nations Department of Economic and Social Affairs, 2017). For instance, a project may assess the change in behavior in an area in which the project activities have taken place as well as in an area in which they have not. Regardless of whether a control is used, confounding factors should be considered and addressed in the project design (Lu, 2014). Our results add to the evidence that recognition gap of the effectiveness on sustainable fishing culture, which provides an understanding, is more necessary for the cooperation between branches in a small-scale community in a post-disaster reconstruction design. What constitutes an effective approach for sustainable marine production will depend on the location of the project and the targeted community (Ahmed \& Glaser, 2016a). Behaviors are more likely to help in the understanding of social norms. How the recognition gap can be bridged between idea and practice in environmental sustainability is another question. We show not only tangible and measureable changes in recognition but also changes in behavior or attitude. Our study suggests that under a vulnerable post-disaster reconstruction process, management of natural capital based on horizontal collaboration among different branches will be effective for sustainable MCE. As such, harmony with a marine ecological environment is important for MCE development for communitybased post-disaster reconstruction.

\section{Acknowledgment}

This work was supported by the JSPS KAKENHI Grant Numbers JP26360062, JP16KK0053, and JP17K02105; Environment Research and Technology Development Fund [S-15-2(3) Predicting and Assessing Natural Capital and Ecosystem Services (PANCES)] of the Ministry of the Environment, Japan; Kurita Water and Environment Foundation [16C003]. Thanks are extended to the members of JF branches, Shizugawa and Utatsu in Minamisanriku.

\section{References}

ASC (2016). Five years after Tsunami, Miyagi Prefecture Fisheries Cooperative Oyster farms celebrate ASC certification. Aquaculture Stewardship Council, 11 April, 2016. https://www.ascaqua.org/news/latest-news/ five-years-after-tsunami-miyagi-prefecture-fisheries-cooperativeoyster-farms-celebrate-asc-certification/ (accessed 20 June, 2018). 
Ahmed, N., \& Glaser, M. (2016). Coastal aquaculture, mangrove deforestation and blue carbon emissions: Is REDD+ a solution? Marine Policy, 66(March), 58-66. https://doi.org/10.1016/j.marpol.2016.01.011

Bianchi, T. S., Allison, M. A., Zhao, J., Li, X., Comeaux, R. S., Feagin, R. A., \& Kulawardhana, R. W. (2013). Historical reconstruction of mangrove expansion in the Gulf of Mexico: Linking climate change with carbon sequestration in coastal wetlands. Estuarine, Coastal and Shelf Science, 119, 7-16. https://doi.org/10.1016/j.ecss.2012.12.007

Dyer, C. L., \& Poggie, J. J. (2005). A Total Capital Approach to the Management of Large Marine Ecosystems: Case Studies of Two Natural Resource Disasters. Large Marine Ecosystems, 13, 111136.

Kay, M. (2006). Community-based natural resource management How knowledge is managed, disseminated and used, 52 .

Lu, Y. (2014). Circular economy development mode of coastal and marine areas in China and its evaluation index research - The example of Qingdao. International Journal Bioautomation, 18(2), 121-130. Retrieved from https://www.scopus.com/inward/record.uri?eid=2-s2.0-84904884419\&partnerID $=40 \& \mathrm{md} 5=\mathrm{f3} 8131 \mathrm{a} 99 \mathrm{~b} 45732 \mathrm{f} 954 \mathrm{a} 565 \mathrm{a} 02575 \mathrm{a} 5 \mathrm{a}$

Marine Debris Program. (2017). Behavior Change Guidance for NOAA Marine Debris Program Marine Debris Prevention Grants, https://marinedebris.noaa.gov/sites/default/files /BehaviorChangeGuidance_Prevention\%20Grants_\%20Oct2017.pdf.

Mei, H., \& Yin, Y. (2009). Studies on marine oil spills and their ecological damage. Journal of the Ocean University of China, 8(3), 312-316. https://doi.org/10.1007/s11802-009-00312-5

Pendleton, L., Donato, D. C., Murray, B. C., Crooks, S., Jenkins, W. A., Sifleet, S., ... Baldera, A. (2012). Estimating Global "Blue Carbon" Emissions from Conversion and Degradation of Vegetated Coastal Ecosystems. PLoS ONE, 7(9). https://doi.org/10.1371/journal.pone.0043542

Qu, Q., Tsai, S.-B., Tang, M., Xu, C., \& Dong, W. (2016). Marine Ecological Environment Management Based on Ecological Compensation Mechanisms. Sustainability, 8(12), 1267. https://doi.org/10.3390/su8121267

Thompson, B. S., Clubbe, C. P., Primavera, J. H., Curnick, D., \& Koldewey, H. J. (2014). Locally assessing the economic viability of blue carbon: A case study from Panay Island, the Philippines. Ecosystem Services, 8, 128-140. https://doi.org/10.1016/j.ecoser.2014.03.004

Wong, B. B. M., \& Candolin, U. (2015). Behavioral responses to changing environments. Behavioral Ecology, 26(3), 665-673. https://doi.org/10.1093/beheco/aru183

World Bank and United Nations Department of Economic and Social Affairs. (2017). The Potential of the Blue Economy: Increasing Long-term Benefits of the Sustainable Use of Marine Resources for Small Island Developing States and Coastal Least Developed Countries, 36.

Worm, B., \& Lenihan, H. S. (2014). Threats to Marine Ecosystems. Overfishing and habitat degradation. Marine Community Ecology and Conservation., 449-476. Retrieved from http://www.bren.ucsb.edu/people/Faculty/documents/lehinan_WormandLenihan2013.pdf 